\title{
POTENTIAL PARAMETERS AND ENERGY DEPENDENCE OF ROTATIONAL ENERGY TRANSFER IN MOLECULAR SYSTEMS
}

\author{
P.M. Agrawal, V. Garg* and K.R. Patidar \\ School of Studies in Physics, Vikram University \\ Ujjain, [M.P.] - 456010, India
}

(Received January 7, 1993; revised version April 9, 1993)

The cross-sections for rotational transitions in a diatornic molecule due to collision with an atom were computed. An empirical relation is obtained that gives the dependence of cross-sections on the energy of the colliding system and the parameters of the interaction potential energy surface. The results are interpreted to investigate the mechanism of the rotational energy transfer process. The cross-sections are found to depend linearly on the torque, range of the potential, and the collision time. Depending on the variation in the location of classical turning point with change in energy, the cross-sections may increase or decrease with energy or cross-section versus energy curve may exhibit maxima or minima.

PACS numbers: $35.20 .-\mathrm{i}$

\section{Introduction}

The study of collision-induced rotational energy transfer (RET) processes in molecular systems is of fundamental importance for several areas [1-4] such as lasers, astrophysics, molecular beam experiments, spin-lattice relaxation of NMR signals, ultrasonic absorption and dispersion, thermal conductivity, shock wave propagation, microwave broadening and intermolecular interaction. In the study of molecular scattering data, it is in fact well known by now that the most detailed information on the relaxation time, anisotropy of the interaction, and other such physical aspects, is provided by state-to-state inelastic cross-sections.

We must, therefore, have a powerful tool of estimating cross-sections. Sometimes it is very useful to have an estimate of a set of cross-sections from a few parameters which can be obtained from some known cross-sections. Scaling and fitting laws such as the power-gap law [5] and the exponential-gap law [6] serve

\footnotetext{
*Permanent address: Department of Physics, Government College, Ajmer - 305001, India.
} 
such purpose. Further, it would also be worth while to arrive at some semi-empirical expressions which give the dependence of cross-sections on the parameters of the intermolecular potential [4, 7]. In our previous work [4] we have investigated the linear dependence of cross-sections on the torque at the classical turning point and the collision time. In this paper we present one empirical relationship, obtained by analysing a few sets of computed cross-sections on a model system. We also discuss the physical explanation in the last section.

\section{Computation of cross-sections}

The computations of cross-sections were performed by using the modified infinite order sudden approximation (IOSAM) given by Agrawal and Raff [8]. The IOSAM is the modification of the well-known infinite order sudden approximation [9] (IOSA) to the solution of the Schrödinger equation. According to this modification [8] $\sigma_{\text {IOSAM }}=\sigma_{\text {IOSA }}\left(T_{\mathrm{f}} / T_{\mathrm{i}}\right)^{1 / 2}$, where $T_{\mathrm{f}}$ and $T_{\mathrm{i}}$ are final and initial translational kinetic energies, respectively.

A system of a homonuclear diatomic molecule and an atom was considered, and the following form of the intermolecular potential was used

with

$$
V(r, \theta)=v(r)\left[1+\sum_{l=2}^{10} a_{l} P_{l}(\cos 0)\right]
$$

$$
v(r)=C \mathrm{e}^{-\alpha r},
$$

where $P$ denotes the Legendre polynomial. Due to symmetry of the homonuclear molecule odd terms in the summation in Eq. (1) would be equal to zero. The masses of the molecule and atom are taken as 28.0 and 4.0 amu respectively. The bond length of the molecule is chosen as $1.0 \AA$.

The computations were performed by using the IOSAM. The phase shifts were computed by using a 10-point Gauss-Mehler quadrature of the WKB phase shift equation [10]. 50 phase shifts were computed for each angle 0 . It is noted for a few sets of calculations that the computed cross-sections do not change if 60 phase shifts are calculated.

\section{Results and discussion}

Table I gives four sets of cross-sections $\sigma(0 \rightarrow l)$, corresponding to four different values of $C$ at total energy $E=0.1 \mathrm{eV}$ and $\alpha=3.5 \AA^{-1}$. In this table and in all the calculations presented in this paper, $a_{2}=a_{4}=a_{6}=a_{8}=a_{10}=0.1$ were taken.

As the magnitude of the torque $(-\partial V / \partial 0)$, acting on the molecule is proportional to $C$, one may expect a strong dependence of cross-sections on $C$. Ilowever, only about $60 \%$ increase in the cross-sections corresponding to the $700 \%$ increase in the value of $C$ is observed.

Table I also lists the corresponding values of $r_{0}^{2} / \sigma$, where $r_{0}$ is the distance of the classical turning point of the atom from the centre of mass of the molecule. $r_{0}$ can be estimated [11] by the relation

$$
v\left(r_{0}\right) \approx E
$$




\section{TABLE I}

Computed cross-sections $\sigma(0 \rightarrow l)$ in $\AA^{2}$ and $r_{0}^{2} / \sigma$ values as a function of $l$, and $C$ in $\mathrm{cV}\left(\alpha=3.5 \AA^{-1}\right.$ and $\left.E=0.1 \mathrm{eV}\right)$.

\begin{tabular}{r|r|r|r|r|r|r|r|r}
\hline \hline$l$ & \multicolumn{4}{|c|}{$\sigma(0 \rightarrow l)$ when $C=$} & \multicolumn{4}{|c}{$r_{0}^{2} / \sigma$ when $C=$} \\
\cline { 2 - 9 } & 100 & 200 & 400 & 800 & 100 & 200 & 400 & 800 \\
\hline 2 & 0.477 & 0.568 & 0.668 & 0.771 & 8.17 & 8.30 & 8.41 & 8.55 \\
4 & 0.251 & 0.300 & 0.351 & 0.407 & 15.51 & 15.72 & 16.00 & 16.20 \\
6 & 0.176 & 0.207 & 0.244 & 0.282 & 22.13 & 22.78 & 23.02 & 23.38 \\
8 & 0.123 & 0.146 & 0.172 & 0.199 & 31.67 & 32.30 & 32.65 & 33.13 \\
10 & 0.080 & 0.095 & 0.110 & 0.127 & 48.69 & 49.64 & 51.05 & 51.91
\end{tabular}

which leads to

$$
r_{0}=\frac{1}{\alpha} \ln (C / E) \text {. }
$$

The values of $r_{0}^{2} / \sigma$ reported in the table show that a $60 \%$ variation in the cross-sections reduces to less than $7 \%$ variation in $r_{0}^{2} / \sigma$. One may thus infer that $r_{0}^{2} / \sigma$ almost remains unchanged with the increase in the potential parameter $C$. Results with $\alpha=3.0 \AA^{-1}$ also show similar behaviour [4].

The calculations were also performed at $E=0.075$ and $0.05 \mathrm{eV}$. The $r_{0}^{2} / \sigma$ and $\sigma$ values are given in Tables II and III. Here also we note that $r_{0}^{2} / \sigma$ values do not change with $C$.

\section{TABLE II}

Computed cross-sections $\sigma(0 \rightarrow l)$ in $\AA^{2}$ and $r_{0}^{2} / \sigma$ values as a function of $l$, and $C$ in $\mathrm{eV}$ $\left(\alpha=3.0 \AA^{-1}\right.$ and $\left.E=0.075 \mathrm{cV}\right)$.

\begin{tabular}{r|r|r|r|r|r|r}
\hline \hline$l$ & \multicolumn{2}{|c|}{$\sigma(0 \rightarrow l)$ when $C=$} & \multicolumn{3}{|c}{$r_{0}^{2} / \sigma$ when $C=$} \\
\cline { 2 - 7 } & 200 & 400 & 800 & \multicolumn{1}{c}{200} & \multicolumn{1}{c}{400} & \multicolumn{1}{c}{800} \\
\hline 2 & 0.838 & 0.980 & 1.126 & 8.25 & 8.35 & 8.49 \\
4 & 0.440 & 0.517 & 0.590 & 15.71 & 15.83 & 16.03 \\
6 & 0.307 & 0.359 & 0.414 & 22.52 & 22.79 & 23.09 \\
8 & 0.217 & 0.253 & 0.290 & 31.86 & 32.34 & 32.96 \\
10 & 0.138 & 0.159 & 0.184 & 50.10 & 51.46 & 51.95
\end{tabular}

This variation, $\sigma$ proportional to $r_{0}^{2}$, may be used to interpret the mechanism of rotational energy transfer process. It can be seen that the torque acting on the molecule at $r_{0}$ is proportional to $v\left(r_{0}\right)$. Further, the collision time may be considered as $\approx 2 r_{0} / v_{\text {rel }}$, where $v_{\text {rel }}$ is the average relative speed. The variation in the effective range of the potential inay be considered proportional to $r_{0}$. From 


\section{TABLE III}

Computed cross-sections $\sigma(0 \rightarrow l)$ in $\AA^{2}$ and $r_{0}^{2} / \sigma$ values as a function of $l$, and $C$ in $\mathrm{eV}$ $\left(\alpha=3.0 \AA^{-1}\right.$ and $\left.E=0.05 \mathrm{eV}\right)$.

\begin{tabular}{r|r|r|r|c|c|c}
\hline \hline$l$ & \multicolumn{2}{|c|}{$\sigma(0 \rightarrow l)$ when $C=$} & \multicolumn{3}{|c}{$r_{0}^{2} / \sigma$ when $C=$} \\
\cline { 2 - 7 } & 200 & 400 & 800 & 200 & 400 & 800 \\
\hline 2 & 0.680 & 0.784 & 0.903 & 11.24 & 11.45 & 11.53 \\
4 & 0.352 & 0.409 & 0.468 & 21.71 & 21.94 & 22.25 \\
6 & 0.241 & 0.278 & 0.320 & 31.71 & 32.28 & 32.54 \\
8 & 0.173 & 0.201 & 0.231 & 44.18 & 44.65 & 45.07 \\
10 & 0.121 & 0.140 & 0.160 & 63.16 & 64.10 & 65.08
\end{tabular}

these, we see that corresponding to a fixed value of $E$ and $\alpha$ the increase in $C$ does not alter the torque acting at $r_{0}$ (see Eq. (3)) but increases $r_{0}$. It would be reasonable to assume that the cross-sections would be proportional to the average torque and the average torque would be proportional to the torque at $r_{0}$. With these considerations the observed variation, $\sigma$ proportional to $r_{0}^{2}$, thus suggests that the cross-sections depend linearly on the range of the potential and the collision time.

The cross-sections corresponding to $\alpha=3.0 \AA^{-1}, E=0.1 \mathrm{eV}$ and $C=200$, 400 and $800 \mathrm{eV}$, are given in Ref. [4]. These data give $r_{0}^{2} / \sigma(0 \rightarrow l)$ values as 6.89, $12.82,18.33,26.53$, and 46.89 for $l=2,4,6,8$ and 10 respectively, for $C=400$. If we compared these $r_{0}^{2} / \sigma$ values with those given in Table I, we would have $\alpha$ dependence. We see that with the increase in $\alpha$ the $r_{0}^{2} / \sigma$ values increase by about a factor equal to the ratio of two values of $\alpha$. The physical explanation of this fact is a matter of further investigation and may be traced by performing quasi-classical trajectory calculations [12] and analysing different dynamical parameters for at least two values of $\alpha$. However, one of the reasons may be such that the average torque is not proportional to the torque at $r_{0}$, i.e., the average torque may depend on $\alpha$.

A comparison of the results reported in Tables II and III, and those given in Ref. [4] at $E=0.1 \mathrm{eV}$ gives the effect of variation of $E$. We see that cross-sections increase with the increase in $E$. Qualitatively, with the increase in $E$ the value of $r_{0}$ as well as collision time decreases, and the value of torque at $r_{0}$ increases. Quantitatively, if we assume the linear dependence of cross-sections on the torque at $r_{0}$, on the range, and on the collision time, then with the variation in $E$ the cross-sections would vary as $r_{0}^{2} E^{x}$. The value of $x$ would be 0.5 if the average relative speed varies as $E^{0.5}$. The present results, however, reveal that the cross-sections vary as $r_{0}^{2} E^{x^{\prime}}$ where $x^{\prime} \approx 0.75$. The difference in $x^{\prime}$ and $x$ may be due to the fact that the average relative speed may not be proportional to $E^{0.5}$, or the average torque may not be proportional to the torque at $r_{0}$. 
Combining the effects of variation of all these three parameters, $C, \alpha$, and $E$, one sees that in the range of present studies

$$
\sigma=A r_{0}^{2} E^{0.75} / \alpha
$$

where $A$ is a constant that does not depend on $C, \alpha$, or $E$. The values of $1 / A$ for 13 sets of cross-sections are given in Table IV. This table shows that except

TABLE IV

The $r_{0}^{2} E^{0.75} / \sigma \alpha$ values as a function of $E, \alpha$ and $C$. The units are the same as given in the previous tables.

\begin{tabular}{r|l|l|l|l|l|l|l|l|l|l|l|l|l}
\hline \hline$E$ & 0.1 & 0.1 & 0.1 & 0.1 & 0.1 & 0.1 & 0.1 & 0.075 & 0.075 & 0.075 & 0.05 & 0.05 & 0.05 \\
$\alpha$ & 3.0 & 3.0 & 3.0 & 3.5 & 3.5 & 3.5 & 3.5 & 3.0 & 3.0 & 3.0 & 3.0 & 3.0 & 3.0 \\
$C$ & 200 & 400 & 800 & 100 & 200 & 400 & 800 & 200 & 400 & 800 & 200 & 400 & 800 \\
\hline$l=2$ & 0.40 & 0.41 & 0.41 & 0.42 & 0.42 & 0.43 & 0.43 & 0.39 & 0.40 & 0.41 & 0.40 & 0.40 & 0.41 \\
4 & 0.75 & 0.76 & 0.78 & 0.79 & 0.80 & 0.81 & 0.82 & 0.75 & 0.76 & 0.77 & 0.77 & 0.77 & 0.78 \\
6 & 1.06 & 1.09 & 1.11 & 1.12 & 1.16 & 1.17 & 1.19 & 1.08 & 1.09 & 1.10 & 1.12 & 1.14 & 1.15 \\
8 & 1.53 & 1.57 & 1.60 & 1.61 & 1.64 & 1.66 & 1.68 & 1.52 & 1.54 & 1.57 & 1.56 & 1.57 & 1.59 \\
10 & 2.72 & 2.78 & 2.84 & 2.47 & 2.52 & 2.59 & 2.64 & 2.39 & 2.46 & 2.48 & 2.23 & 2.26 & 2.29
\end{tabular}

for the fair agreement seen among numbers in the last row of the table, Eq. (5) is found to be in good to excellent agreement for all other rows of the table. The calculations were also attempted for many other values of $a_{l}$ in the range 0.01 to 0.1 . The validity of Eq. (5) was noted for all the cases.

Equation (5) can be used as a scaling law to compute the cross-sections at some values of $E, \alpha$, and $C$ from the known cross-sections at some other values of $E, \alpha$, and $C$. As an example, the cross-sections at $E=0.091 \mathrm{eV}, C=500 \mathrm{eV}$ and

\section{TABLE V}

Cross-sections in $\AA^{2}$ computed by IOSAM and those obtained using Eq. (5). $E=0.091 \mathrm{eV}$, $\alpha=3.23 \AA^{-1}$ and $C=500 \mathrm{eV}$.

\begin{tabular}{r|c|c}
\hline \hline$l$ & \multicolumn{2}{|c}{$\sigma(0 \rightarrow l)$} \\
\cline { 2 - 3 } & IOSAM & Eq. $(5)$ \\
\hline 2 & 0.876 & 0.911 \\
4 & 0.463 & 0.474 \\
6 & 0.323 & 0.326 \\
8 & 0.228 & 0.234 \\
10 & 0.141 & 0.163
\end{tabular}

$\alpha=3.23 \AA^{-1}$ were computed by using IOSAM and are compared in Table V with those given by Eq. (5) and the cross-sections at $E=0.05 \mathrm{eV}$ and $\alpha=3.0 \AA^{-1}$ (Table III). An excellent agreement between the two sets of cross-sections shows the usefulness of Eq. (5). 
Equation (5) shows that with the change in $E$ the cross-sections vary as the product of two factors, $r_{0}^{2}$ and $E^{0.75}$. The former factor decreases and the latter increases with the increase in $E$. Thus according to the relative variation of these two factors the cross-sections may increase or decrease with the increase in energy, or may exhibit maxima or minima in $\sigma$ vs. $E$ curve. It may be mentioned that the experimental data given by Barnes et al. [13], for IIF-Ne, $\mathrm{Ar}$, and $\mathrm{Kr}$ systems show all such possible variations of $\sigma$ with $E$. Theoretical results on different systems also show different behaviour as regards the variation in $\sigma$ with $E$. The close coupling calculations by Allison and Dalgarno [14] on $\mathrm{II}_{2}-\mathrm{IIe}$ show increase in $\sigma$ with the increase in $E$, whereas the computations by Agrawal and Raff [15] on $\mathrm{CO}_{2}-\mathrm{He}$ system show reverse trend. All such different patterns of variations of $\sigma$ with $E$ are, at least qualitatively, consistent with Eq. (5). A detailed quantitative study to explain the observed variation of $\sigma$ with $E$ and other parameters is under progress.

\section{References}

[1] M. Faubel, Adv. At. Mol. Phys. 10, 345 (1983).

[2] A.J. McCaffery, Z.T. Alwahabi, Phys. Rev. A 43, 611 (1991).

[3] P.M. Agrawal, N.C. Agrawal, J. Chem. Phys. 83, 4444 (1985).

[4] P.M. Agrawal, V. Garg, Curr. Sci. 61, 43 (1991).

[5] T.A. Brunner, N. Smith, A.W. Karp, D.E. Pritchard, J. Chem. Phys. 74, 3324 (1981).

[6] J.C. Polanyi, K.B. Woodall, J. Chem. Phys. 56, 1563 (1972).

[7] L. Eno, H. Rabitz, J. Chem. Phys. 72, 2314 (1980).

[8] P.M. Agrawal, L.M. Raff, J. Chem. Phys. 74, 3292 (1981).

[9] G.A. Parker, R.T. Pack, J. Chem. Phys. 68, 1585 (1978).

[10] R.T. Pack, J. Chem. Phys. C0, 633 (1974).

[11] S.L. Dexheimer, M. Durand, T.A. Brunner, D.E. Pritclard, J. Chem. Phys. 7G, 4996 (1982).

[12] L.M. Raff, D.L. Thompson, in: Theory of Chemical Reaction Dynamics, Vol. III, Ed. M. Baer, Chemical Rubber, Boca Raton 1985; P.M. Agrawal, D.L. Thompson, L.M. Raft, J. Chem. Phys. 92, 1069 (1990).

[13] J.A. Barnes, M. Keil, R.E. Kutina, J.C. Polanyi, J. Chem. Phys. 76, 913 (1982).

[14] A.C. Allison, A. Dalgarno, Proc. Phys. Soc. 00, 609 (1967).

[15] P.M. Agrawal, L.M. Rafl, J. Chem. Phys. 75, 2163 (1981). 